\title{
Stable Robin solid wall boundary conditions for the Navier-Stokes equations
}

\author{
Jonas Berg and Jan Nordström
}

\section{Linköping University Post Print}

\section{N.B.: When citing this work, cite the original article.}

Original Publication:

Jonas Berg and Jan Nordström, Stable Robin solid wall boundary conditions for the NavierStokes equations, 2011, Journal of Computational Physics, (230), 19, 7519-7532.

http://dx.doi.org/10.1016/j.jcp.2011.06.027

Copyright: Elsevier

http://www.elsevier.com/

Postprint available at: Linköping University Electronic Press

http://urn.kb.se/resolve?urn=urn:nbn:se:liu:diva-70019 


\title{
Stable Robin Solid Wall Boundary Conditions for the Navier-Stokes Equations
}

\author{
Jens Berg* \\ Uppsala University, Department of Information Technology, SE-751 05, Uppsala, Sweden \\ Jan Nordström \\ Linköping University, Department of Mathematics, SE-581 83, Linköping, Sweden
}

\begin{abstract}
In this paper we prove stability of Robin solid wall boundary conditions for the compressible Navier-Stokes equations. Applications include the no-slip boundary conditions with prescribed temperature or temperature gradient and the first order slip-flow boundary conditions. The formulation is uniform and the transitions between different boundary conditions are done by a change of parameters. We give different sharp energy estimates depending on the choice of parameters.

The discretization is done using finite differences on Summation-By-Parts form with weak boundary conditions using the Simultaneous Approximation Term. We verify convergence by the method of manufactured solutions and show computations of flows ranging from no-slip to almost full slip.
\end{abstract}

Keywords: Navier-Stokes, Robin boundary conditions, Well-posedness, Stability, High order accuracy, Summation-By-Parts

\section{Introduction}

There has recently been a development of stable boundary [1,2] and interface [3] conditions of a specific form for the compressible Navier-Stokes equations. This paper extends the result in [2] to more general solid wall boundary conditions and includes sharp energy estimates. While [2] deals only with the no-slip boundary conditions, we will provide a uniform formulation which includes the no-slip boundary conditions with prescribed temperature or temperature gradient and slip-flow boundary conditions or any combination thereof.

The tools that we will use to obtain a uniform formulation together with proof of stability are finite difference approximations on Summation-By-Parts (SBP) form together with the Simultaneous Approximation term. This method has the benefit

*Corresponding author: Jens Berg

Address: Division of Scientific Computing, Department of Information Technology, Uppsala University, Box 337, SE-751 05, Uppsala, Sweden

Phone: +46 18-4716253

Fax: +46 $18523049,+4618511925$

E-mail: jens.berg@it.uu.se 
of being stable by construction for any linear well-posed Cauchy problem $[4,5]$ and the robustness has been shown in a wide range of applications $[5,6,7,8,9]$.

The first derivative is approximated by $u_{x} \approx D v=P^{-1} Q v$, where $v$ is the discrete grid function, $D$ is the differentiation matrix, $P=P^{T}>0$ defines a norm by $\|v\|^{2}=v^{T} P v$ and $Q$ has the SBP property $Q+Q^{T}=B=[-1,0, \ldots, 0,1]^{T}$. See $[10,11]$ for details about these operators.

There exist operators accurate of order 2, 4, 6 and 8 and the stability analysis does not depend on the order of accuracy of the operators. We will pose our equations on conservative form and hence we do not need an operator approximating the second derivative. Operators approximating the second derivative with constant coefficients are derived in [11] and have recently been developed for variable coefficients problems in [12].

The boundary conditions will be imposed weakly using the Simultaneous Approximation Term (SAT). The SAT term is added to the right-hand-side of the discretized equations as a penalty term which forces the equation towards the boundary conditions. Together the SBP and SAT technique provide a tool for creating stable approximations for well-posed initial-boundary value problems. The relation between weak and strong boundary conditions in terms of accuracy is discussed in $[13]$.

\section{The Navier-Stokes equations}

\subsection{Continuous case}

We consider the two-dimensional Navier-Stokes equations on conservative form

$$
q_{t}+F_{x}+G_{y}=0
$$

where

$$
F=F^{I}-\varepsilon F^{V}, \quad G=G^{I}-\varepsilon G^{V} .
$$

The superscript $I$ denotes the inviscid part of the fluxes and $V$ the viscous part. The components of the solution vector are $q=[\rho, \rho u, \rho v, e]^{T}$ which are the density, $\mathrm{x}$ - and $\mathrm{y}$-directional momentum respectively and energy. The components of the fluxes are given by

$$
\begin{aligned}
F^{I} & =\left[\rho u, p+\rho u^{2}, \rho u v, u(p+e)\right]^{T} \\
G^{I} & =\left[\rho v, \rho u v, p+\rho v^{2}, v(p+e)\right]^{T} \\
F^{V} & =\left[0, \tau_{x x}, \tau_{x y}, u \tau_{x x}+v \tau_{x y}-Q_{x}\right]^{T} \\
G^{V} & =\left[0, \tau_{x y}, \tau_{y y}, u \tau_{y x}+v \tau_{y y}-Q_{y}\right]^{T}
\end{aligned}
$$

where $p$ is the pressure, $\operatorname{Pr}$ the Prandtl number, $\gamma$ the ratio of specific heat and $Q=-\kappa T$ is the thermal conductivity times the temperature according to Fourier's law. The stress tensors are given by

$$
\tau_{x x}=2 \mu \frac{\partial u}{\partial x}+\lambda\left(\frac{\partial u}{\partial x}+\frac{\partial v}{\partial y}\right), \tau_{y y}=2 \mu \frac{\partial v}{\partial y}+\lambda\left(\frac{\partial u}{\partial x}+\frac{\partial v}{\partial y}\right), \tau_{x y}=\tau_{y x}=\mu\left(\frac{\partial u}{\partial y}+\frac{\partial v}{\partial x}\right)
$$


where $\mu$ and $\lambda$ are the dynamic and second viscosity respectively.

All the equations above have been non-dimensionalized as

$$
\begin{gathered}
u=\frac{u^{*}}{c_{\infty}^{*}}, \quad v=\frac{v^{*}}{c_{\infty}^{*}}, \quad \rho=\frac{\rho^{*}}{\rho_{\infty}^{*}}, \quad T=\frac{T^{*}}{T_{\infty}^{*}}, \\
p=\frac{p^{*}}{\rho_{\infty}^{*}\left(c_{\infty}^{*}\right)^{2}}, \quad e=\frac{e^{*}}{\rho_{\infty}^{*}\left(c_{\infty}^{*}\right)^{2}}, \quad \lambda=\frac{\lambda^{*}}{\mu_{\infty}^{*}}, \quad \mu=\frac{\mu^{*}}{\mu_{\infty}^{*}}
\end{gathered}
$$

where the $*$-superscript denotes a dimensional variable and the $\infty$-subscript the freestream value. In (2) we have $\varepsilon=\frac{M a}{R e}$ where $M a$ is the Mach-number and $R e=\frac{\rho_{\infty}^{*} u_{\infty}^{*} L_{\infty}^{*}}{\mu_{\infty}^{*}}$ is the Reynolds-number with $L_{\infty}^{*}$ being a characteristic length scale.

The equations as stated in (1) is a highly non-linear system of equations. The well-posedness and stability conditions that will be derived in this paper will be based on a linear symmetric formulation.

We freeze the coefficients at some constant state $\bar{w}=[\bar{\rho}, \bar{u}, \bar{v}, \bar{p}]^{T}$ and linearize as $\tilde{w}=\bar{w}+w^{\prime}$ where $w^{\prime}=[\rho, u, v, p]^{T}$ is a perturbation around the constant state $\bar{w}$. This yields an equation with constant matrix coefficients. Next we transform to primitive variables and use the parabolic symmetrizer derived in [14] to get the linear, constant coefficient and symmetric system

$$
w_{t}+A w_{x}+B w_{y}=\varepsilon\left(\left(C_{11} w_{x}+C_{12} w_{y}\right)_{x}+\left(C_{21} w_{x}+C_{22} w_{y}\right)_{y}\right)
$$

where the symmetrized variables are

$$
w=\left[\frac{\bar{c}}{\sqrt{\gamma} \bar{\rho}} \rho, u, v, \frac{1}{\bar{c} \sqrt{\gamma(\gamma-1)}} T\right]^{T} .
$$

All matrix coefficients can be found in [14] but we restate them in Appendix A for convenience.

We will use the energy method to determine the well-posedness of (6). The energy norm in which we will derive our estimates is defined by

$$
\|w\|^{2}=\int_{\Omega} w^{T} w d \Omega .
$$

By multiplying (6) with $w^{T}$, integrating over $\Omega$ and using the Gauss-Green theorem for higher-dimensional integration by parts we obtain

$$
\begin{aligned}
\|w\|_{t}^{2} & =-\oint_{\partial \Omega} w^{T}\left(A w-2 \varepsilon\left(C_{11} w_{x}+C_{12} w_{y}\right), B w-2 \varepsilon\left(C_{21} w_{x}+C_{22} w_{y}\right)\right) \cdot n d s \\
& -2 \varepsilon \int_{\Omega}\left[\begin{array}{l}
w_{x} \\
w_{y}
\end{array}\right]^{T}\left[\begin{array}{ll}
C_{11} & C_{12} \\
C_{21} & C_{22}
\end{array}\right]\left[\begin{array}{l}
w_{x} \\
w_{y}
\end{array}\right] d \Omega
\end{aligned}
$$

where the last term in (9) can be seen to be dissipative by computing the eigenvalues of the matrix in the quadratic form $[1,2,4]$. 
To simplify we let the domain of interest be the unit square $0 \leq x, y \leq 1$ and we consider only the south boundary at $y=0$. Equation (9) is then simplified to

$$
\begin{aligned}
\|w\|_{t}^{2} & =\left.\int_{0}^{1} w^{T}\left(B w-2 \varepsilon\left(C_{21} w_{x}+C_{22} w_{y}\right)\right)\right|_{y=0} d x \\
& -2 \varepsilon \int_{\Omega}\left[\begin{array}{l}
w_{x} \\
w_{y}
\end{array}\right]^{T}\left[\begin{array}{ll}
C_{11} & C_{12} \\
C_{21} & C_{22}
\end{array}\right]\left[\begin{array}{l}
w_{x} \\
w_{y}
\end{array}\right] d \Omega .
\end{aligned}
$$

The east, west and north boundaries are omitted and we consider the south boundary as a solid wall.

A solid wall requires 3 boundary conditions $[2,4]$. Since we do not want any penetration through the wall we require that

$$
v(x, 0, t)=0 .
$$

A Robin boundary condition does not apply to the $v$-velocity since it is not a wellposed boundary condition for the Euler equations. When inserting (11) into (10) and considering only the south boundary at $y=0$ we get

$$
\|w\|_{t}^{2} \leq-2 \varepsilon \int_{0}^{1}\left(\frac{\mu}{\bar{\rho}} u u_{y}+\frac{\gamma \mu}{\bar{\rho} \bar{c}^{2} \gamma(\gamma-1) \operatorname{Pr}} T T_{y}\right) d x .
$$

Note that the dissipative term has been omitted and the equality has been replaced by an inequality.

We are allowed to use 2 more boundary conditions. The boundary conditions we consider are the Robin conditions

$$
\alpha u-\beta u_{y}=g_{1}, \quad \phi T-\psi T_{y}=g_{2},
$$

where any combiation of $\alpha, \beta, \phi$ and $\psi$ are allowed as long as no boundary condition is removed. This allows us to study all physically relevant boundary conditions in one uniform formulation. In particular we can include the standard no-slip boundary conditions with prescribed temperature or temperature gradient and the first order slip-flow boundary conditions.

Remark 2.1. Note that if $u(x, 0, t) \neq 0$ then we need to use that $v(x, 0, t)=0$ imply $v_{x}(x, 0, t)=0$ to obtain (12). As we shall see later, the relation $v_{x}(x, 0, t)=0$ must be explicitly included in the discrete case in order to obtain stability.

Depending on how we chose $\alpha, \beta, \phi$ and $\psi$ in (13) we obtain different energy estimates. Assume that $g_{1,2}=0$. If we restrict ourselves to the case where $\beta, \psi \neq 0$ and insert (13) into (12) we obtain the energy estimate

$$
\|w\|_{t}^{2} \leq-2 \varepsilon \int_{0}^{1}\left(\frac{\mu}{\bar{\rho}} \frac{\alpha}{\beta} u^{2}+\frac{\gamma \mu}{\bar{\rho} \bar{c}^{2} \gamma(\gamma-1) \operatorname{Pr}} \frac{\phi}{\psi} T^{2}\right) d x .
$$

We can see that the energy is bounded if

$$
\alpha \beta \geq 0, \quad \phi \psi \geq 0 \text {. }
$$


We can now let $\alpha, \phi \rightarrow 0$ and obtain the Neumann boundary conditions which have the energy estimate $\|w\|_{t}^{2} \leq 0$. By restricting ourselves to the case where $\alpha, \phi \neq 0$ we get the energy estimate

$$
\|w\|_{t}^{2} \leq-2 \varepsilon \int_{0}^{1}\left(\frac{\mu}{\bar{\rho}} \frac{\beta}{\alpha} u_{y}^{2}+\frac{\gamma \mu}{\bar{\rho} \bar{c}^{2} \gamma(\gamma-1) \operatorname{Pr}} \frac{\psi}{\phi} T_{y}^{2}\right) d x
$$

which gives an energy estimate if (15) hold. If we let $\beta, \psi \rightarrow 0$ we recover the standard no-slip boundary conditions which have the energy estimate $\|w\|_{t}^{2} \leq 0$. Compared to the Robin boundary conditions (13), the no-slip boundary conditions are less damping than if we keep $\alpha, \beta, \phi$ and $\psi$ non-zero.

\subsection{Discrete case}

To extend the SBP and SAT technique to systems in higher dimensions it is convenient to introduce the Kronecker product, which is defined for arbitrary matrices $A \in \mathbb{R}^{m \times n}$ and $B \in \mathbb{R}^{p \times q}$ by

$$
A \otimes B=\left[\begin{array}{ccc}
a_{11} B & \cdots & a_{1 n} B \\
\vdots & \ddots & \vdots \\
a_{m 1} B & \cdots & a_{m n} B
\end{array}\right] .
$$

As a special case of a tensor product, the Kronecker product is bilinear and associative, and one can prove the mixed product property $(A \otimes B)(C \otimes D)=(A C \otimes B D)$ if the usual matrix products are defined. For inversion and transposing we have

$$
(A \otimes B)^{-1, T}=\left(A^{-1, T} \otimes B^{-1, T}\right)
$$

if the usual inverse exist. The mixed product property is particularly useful since it allows the operators to operate in each coordinate direction independently of each other.

Let the domain $0 \leq x, y \leq 1$ be discretized by $M+1$ and $N+1$ equidistant grid points respectively. We define the following operators:

$$
D_{x}=P_{x}^{-1} Q_{x}, \quad D_{y}=P_{y}^{-1} Q_{y}, \quad Q_{x, y}+Q_{x, y}^{T}=B_{x, y}=\operatorname{diag}(-1,0, \ldots, 0,1)
$$

where $P_{x, y}$ is symmetric and positive definite. In this paper a diagonal $P_{x, y}$ is used but there are more general forms available [10,11]. The details for the second order case are found in Appendix B.

The extension to the two-dimensional domain is done using the Kronecker product. The following matrices will be used:

$$
\begin{array}{lll}
\bar{D}_{x}=\left(D_{x} \otimes I_{y} \otimes I_{4}\right), & \bar{D}_{y}=\left(I_{x} \otimes D_{y} \otimes I_{4}\right), & \bar{P}_{x}=\left(P_{x} \otimes I_{y} \otimes I_{4}\right) \\
\bar{P}_{y}=\left(I_{x} \otimes P_{y} \otimes I_{4}\right) & \bar{P}=\left(P_{x} \otimes P_{y} \otimes I_{4}\right) & \bar{B}_{x}=\left(B_{x} \otimes I_{y} \otimes I_{4}\right) \\
\bar{B}_{y}=\left(I_{y} \otimes B_{y} \otimes I_{4}\right) & \bar{C}_{11}=\left(I_{x} \otimes I_{y} \otimes C_{11}\right) & \bar{C}_{12}=\left(I_{x} \otimes I_{y} \otimes C_{12}\right) \\
\bar{C}_{21}=\left(I_{x} \otimes I_{y} \otimes C_{21}\right) & \bar{C}_{22}=\left(I_{x} \otimes I_{y} \otimes C_{22}\right) & \bar{E}_{0}=\left(I_{x} \otimes E_{0} \otimes I_{4}\right)
\end{array}
$$

where $E_{0}=\operatorname{diag}(1,0, \ldots, 0)$. The solution vector is aligned as $w=\left[w_{0}, \ldots, w_{M \times N}\right]^{T}=$ $\left[\rho_{0},(\rho u)_{0},(\rho v)_{0}, e_{0}, \ldots, \rho_{M \times N},(\rho u)_{M \times N},(\rho v)_{M \times N}, e_{M \times N}\right]^{T}$. 
With the definitions given in (20) we can discretize (1) as

$$
w_{t}+\bar{D}_{x} \mathbf{F}+\bar{D}_{y} \mathbf{G}=0
$$

where $w, \mathbf{F}$ and $\mathbf{G}$ are the discrete grid function and fluxes. In order to analyze (21) we need to use the linear, symmetric formulation (6). After linearizing, freezing the coefficients and transforming to symmetric variables, we apply the energy method to (21) by multiplying with $w^{T} \bar{P}$ and using the SBP properties of the operators. For a thorough derivation, see $[1,4]$. The result is

$$
\begin{aligned}
\|w\|_{t}^{2} & +w^{T} \bar{B}_{x} \bar{P}_{y}\left(\mathbf{F}_{s}^{I}-2 \varepsilon \mathbf{F}_{s}^{V}\right)+w^{T} \bar{B}_{y} \bar{P}_{x}\left(\mathbf{G}_{s}^{I}-2 \varepsilon \mathbf{G}_{s}^{V}\right) \\
& +2 \varepsilon\left[\begin{array}{c}
\bar{D}_{x} w \\
\bar{D}_{y} w
\end{array}\right]^{T}\left[\begin{array}{cc}
\bar{P} & 0 \\
0 & \bar{P}
\end{array}\right]\left[\begin{array}{ll}
\bar{C}_{11} & \bar{C}_{12} \\
\bar{C}_{21} & \bar{C}_{22}
\end{array}\right]\left[\begin{array}{c}
\bar{D}_{x} w \\
\bar{D}_{y} w
\end{array}\right]=0 .
\end{aligned}
$$

where the norm is defined by $\|w\|^{2}=w^{T} \bar{P} w$ and

$$
\begin{aligned}
& \mathbf{F}_{s}^{I}=\bar{A} w, \quad \mathbf{F}_{s}^{V}=\bar{C}_{11} w_{x}+\bar{C}_{12} w_{y} \\
& \mathbf{G}_{s}^{I}=\bar{B} w, \quad \mathbf{G}_{s}^{V}=\bar{C}_{21} w_{x}+\bar{C}_{22} w_{y}
\end{aligned}
$$

with $\bar{A}=\left(I_{x} \otimes I_{y} \otimes A\right)$ and $\bar{B}=\left(I_{x} \otimes I_{y} \otimes B\right)$. The last term in (22) is dissipative and we need to construct a SAT which bounds the indefinite boundary terms.

To simplify we consider only the terms related to the south boundary at $y=0$. Equation (22) becomes

$$
\|w\|_{t}^{2}-w^{T} \bar{P}_{x} \bar{E}_{0}\left(\mathbf{G}_{s}^{I}-2 \varepsilon \mathbf{G}_{s}^{V}\right)+2 \varepsilon\left[\begin{array}{c}
\bar{D}_{x} w \\
\bar{D}_{y} w
\end{array}\right]^{T}\left[\begin{array}{cc}
\bar{P} & 0 \\
0 & \bar{P}
\end{array}\right]\left[\begin{array}{ll}
\bar{C}_{11} & \bar{C}_{12} \\
\bar{C}_{21} & \bar{C}_{22}
\end{array}\right]\left[\begin{array}{c}
\bar{D}_{x} w \\
\bar{D}_{y} w
\end{array}\right]=0 .
$$

Denote the last term in (24) by $D I$ and expand the fluxes according to the the definitions in (23). Equation (24) then simplifies to

$$
\|w\|_{t}^{2}-\underbrace{w^{T} \bar{P}_{x} \bar{E}_{0} \bar{B} w+2 \varepsilon w^{T} \bar{P}_{x} \bar{E}_{0}\left(\bar{C}_{21} w_{x}+\bar{C}_{22} w_{y}\right)}_{\mathrm{BT}}+D I=0 .
$$

Based on (25) we will construct a SAT which we add to the right-hand-side of (21) that will bound the indefinite terms and implement the correct boundary conditions.

Remember that the boundary conditions being imposed are

$$
\alpha u-\beta u_{y}=g_{1}, \quad \phi T-\psi T_{y}=g_{2}, \quad v=g_{3}
$$

where $g_{3}$ will be set to zero at a solid wall. In order to obtain stability we also need to include the discrete version of

$$
v_{x}=\frac{\partial g_{3}}{\partial x}
$$

which does not automatically follow from (26) as it does in the continuous case. 
Due to the different forms of the boundary conditions we split the SAT into 5 different terms. One term for the inviscid part and one additional term for each condition in (26) and (27). The SAT we will use is

$$
\begin{aligned}
\mathbb{S} & =\bar{P}_{y}^{-1} \bar{E}_{0} \bar{\Sigma}\left(w-g^{I}\right) \\
& +\varepsilon \sigma_{2} \bar{P}_{y}^{-1} \bar{E}_{0}\left(\alpha \bar{H}_{2} w-\beta \bar{D}_{y} \bar{H}_{2} w-g_{1}\right) \\
& +\varepsilon \sigma_{3} \bar{P}_{y}^{-1} \bar{E}_{0}\left(\bar{H}_{3} w-g_{3}\right)+\varepsilon \bar{P}_{y}^{-1} \bar{E}_{0} \bar{\Theta}\left(\bar{D}_{x} w-\frac{\partial g_{3}}{\partial x}\right) \\
& +\varepsilon \sigma_{4} \bar{P}_{y}^{-1} \bar{E}_{0}\left(\phi \bar{H}_{4} w-\psi \bar{D}_{y} \bar{H}_{4} w-g_{2}\right)
\end{aligned}
$$

where $\bar{H}_{i}=\left(I_{x} \otimes I_{y} \otimes H_{i}\right)$ and $H_{i}$ are $4 \times 4$ matrices that have the only nonzero element 1 at the $(i, i)$ position on the diagonal. We have $\bar{\Sigma}=\left(I_{x} \otimes I_{y} \otimes \Sigma\right)$ where $\Sigma$ is an undetermined $4 \times 4$ matrix that will be determined for stability. $\bar{\Theta}=\left(I_{x} \otimes I_{y} \otimes \Theta\right)$ where $\Theta$ is a $4 \times 4$ penalty matrix that acts on $v$ only and hence has the structure

$$
\Theta=\left[\begin{array}{llll}
0 & 0 & \theta_{1} & 0 \\
0 & 0 & \theta_{2} & 0 \\
0 & 0 & \theta_{3} & 0 \\
0 & 0 & \theta_{4} & 0
\end{array}\right]
$$

Both $\bar{\Sigma}$ and $\bar{\Theta}$ will be determined for stability.

The first row in (28) is used to bound the inviscid part and the three last rows are scaled with $\varepsilon$ and enforces each of the boundary conditions in (26) and (27). This construction will ensure that the solution converges to that of the Euler equations as $\varepsilon \rightarrow 0$. The Robin boundary conditions does not apply to the Euler equations. Hence as $\varepsilon \rightarrow 0$, the viscous terms mush vanish and leave $v=0$ as the only boundary condition for the Euler equations at a solid wall.

By considering zero boundary data and carrying (28) through the derivations in the energy estimate it will appear on the right-hand-side of (24) as

$$
\begin{aligned}
2 w^{T} \bar{P} \mathbb{S} & =2 w^{T} \bar{P}_{x} \bar{E}_{0} \bar{\Sigma} w \\
& +2 \varepsilon \sigma_{2} w^{T} \bar{P}_{x} \bar{E}_{0}\left(\alpha \bar{H}_{2} w-\beta \bar{D}_{y} \bar{H}_{2} w\right) \\
& +2 \varepsilon \sigma_{3} w^{T} \bar{P}_{x} \bar{E}_{0} \bar{H}_{3} w+2 \varepsilon \bar{P}_{x} \bar{E}_{0} \bar{D}_{x} \bar{\Theta} w \\
& +2 \varepsilon \sigma_{4} w^{T} \bar{P}_{x} \bar{E}_{0}\left(\phi \bar{H}_{4} w-\psi \bar{D}_{y} \bar{H}_{4} w\right) .
\end{aligned}
$$

By moving all terms to the right hand side we get

$$
\|w\|_{t}^{2}=\mathrm{BT}+\mathrm{SAT}-\mathrm{DI}
$$

and we have to choose the coefficients in (28) such that $\|w\|_{t}^{2} \leq 0$. In order to proceed we split the BT and SAT into inviscid and viscous parts respectively.

By considering only the inviscid terms we have

$$
\begin{aligned}
\|w\|_{t}^{2} & =w^{T} \bar{P}_{x} \bar{E}_{0} \bar{B} w+2 w^{T} \bar{P}_{x} \bar{E}_{0} \bar{\Sigma} w \\
& =w^{T} \bar{P}_{x} \bar{E}_{0}(\bar{B}+2 \bar{\Sigma}) w
\end{aligned}
$$

and we have to choose $\bar{\Sigma}$ such that $\bar{B}+2 \bar{\Sigma} \leq 0$. Since the Kronecker product preserves positive semi-definiteness it is sufficient to determine the $4 \times 4$ matrix $\Sigma$ such that

$$
B+2 \Sigma \leq 0
$$


This is easily accomplished by diagonalizing $B=X \Lambda X^{T}$ and rewriting (34) as

$$
\Lambda^{+}+\Lambda^{-}+2 \Sigma_{c} \leq 0
$$

where $\Lambda^{+,-}$holds the positive and non-positive eigenvalues of $B$ respectively and $\Sigma_{c}=X^{T} \Sigma X$. We have $\Lambda^{+}=\operatorname{diag}(0,0, \bar{c}, 0)$ and hence we construct $\Sigma_{c}=\operatorname{diag}(0,0, \sigma, 0)$ with $\sigma \leq-\frac{\bar{c}}{2}$. By transforming back we get

$$
\Sigma=X \Sigma_{c} X^{T}=\frac{\sigma}{2 \gamma}\left[\begin{array}{cccc}
1 & 0 & \sqrt{\gamma} & \sqrt{\gamma(\gamma-1)} \\
0 & 0 & 0 & 0 \\
\sqrt{\gamma} & 0 & \gamma & \sqrt{\gamma(\gamma-1)} \\
\sqrt{\gamma(\gamma-1)} & 0 & \sqrt{\gamma(\gamma-1)} & \gamma-1
\end{array}\right]
$$

With $\Sigma$ given by (36) the inviscid boundary terms are bounded and implements the wall normal velocity boundary condition for the Euler equations.

Remark 2.2. The transformation from conservative to primitive to symmetric to characteristic variables and back is used only for the purpose of analysis. In a code the transformation from conservative to characteristic variables and back can be done directly by following the transformations given in [15].

By considering only the viscous terms we have

$$
\begin{aligned}
\|w\|_{t}^{2} & =-2 \varepsilon w^{T} \bar{P}_{x} \bar{E}_{0}\left(\bar{D}_{x} \bar{C}_{21} w+\bar{D}_{y} \bar{C}_{22} w\right) \\
& +2 \varepsilon \sigma_{2} w^{T} \bar{P}_{x} \bar{E}_{0}\left(\alpha \bar{H}_{2} w-\beta \bar{D}_{y} \bar{H}_{2} w\right) \\
& +2 \varepsilon \sigma_{3} w^{T} \bar{P}_{x} \bar{E}_{0}\left(\bar{H}_{3} w\right)+2 \varepsilon \bar{P}_{x} \bar{E}_{0} \bar{D}_{x} \bar{\Theta} w \\
& +2 \varepsilon \sigma_{4} w^{T} \bar{P}_{x} \bar{E}_{0}\left(\phi \bar{H}_{4} w-\psi \bar{D}_{y} \bar{H}_{4} w\right) \\
& -D I
\end{aligned}
$$

which can be written as a quadratic form

$$
\|w\|_{t}^{2}=-\varepsilon W^{T} \bar{P}_{0} \bar{M}_{0} W-D I
$$

where

$$
W=\left[\begin{array}{c}
w \\
\bar{D}_{x} w \\
\bar{D}_{y} w
\end{array}\right], \quad \bar{P}_{0}=\left[\begin{array}{ccc}
\bar{P}_{x} \bar{E}_{0} & 0 & 0 \\
0 & \bar{P}_{x} \bar{E}_{0} & 0 \\
0 & 0 & \bar{P}_{x} \bar{E}_{0}
\end{array}\right], \quad \bar{M}_{0}=\left[\begin{array}{ccc}
\bar{m}_{1} & \bar{m}_{2} & \bar{m}_{3} \\
\bar{m}_{2}^{T} & 0 & 0 \\
\bar{m}_{3} & 0 & 0
\end{array}\right]
$$

where $\bar{m}_{3}$ and $\bar{M}_{0}$ are symmetric and

$$
\begin{aligned}
& \bar{m}_{1}=-2\left(I_{x} \otimes I_{y} \otimes \sigma_{2} \alpha H_{2}+\sigma_{3} H_{3}+\sigma_{4} \phi H_{4}\right) \\
& \bar{m}_{2}=\left(I_{x} \otimes I_{y} \otimes C_{21}-\Theta\right) \\
& \bar{m}_{3}=\left(I_{x} \otimes I_{y} \otimes C_{22}+\sigma_{2} \beta H_{2}+\sigma_{4} \psi H_{4}\right) .
\end{aligned}
$$

In order to stabilize the viscous terms we need to choose our coefficients $\sigma_{2,3,4}$ and $\Theta$ such that $\bar{M}_{0} \geq 0$. Note that only the positive semi-definiteness of $\bar{M}_{0}$ is required since $\bar{P}_{0}$ is positive semi-definite and commutes with $\bar{M}_{0}$. Hence if $\bar{M}_{0}$ is positive semi-definite, so is the product $\bar{P}_{0} \bar{M}_{0}$. 
Unfortunately though, there is no choice of $\sigma_{2,3,4}$ and $\Theta$ such that $\bar{m}_{1}=\bar{m}_{2}=$ $\bar{m}_{3}=0$ which would give $\bar{M}_{0}=0$. Hence in the current form we will always end up with an indefinite $\bar{M}_{0}$.

To remedy this fact we can use a part of the dissipation term $D I$ in (38),

$$
D I=2 \varepsilon\left[\begin{array}{c}
\bar{D}_{x} w \\
\bar{D}_{y} w
\end{array}\right]^{T}\left[\begin{array}{cc}
\bar{P} & 0 \\
0 & \bar{P}
\end{array}\right]\left[\begin{array}{ll}
\bar{C}_{11} & \bar{C}_{12} \\
\bar{C}_{21} & \bar{C}_{22}
\end{array}\right]\left[\begin{array}{l}
\bar{D}_{x} w \\
\bar{D}_{y} w
\end{array}\right] .
$$

The matrix $\bar{P}$ can be rewritten as

$$
\bar{P}=\left(P_{x} \otimes P_{y} \otimes I_{4}\right)=\left(P_{x} \otimes \tilde{P}_{y}+r E_{0} \otimes I_{4}\right)=\underbrace{\left(P_{x} \otimes \tilde{P}_{y} \otimes I_{4}\right)}_{\tilde{P}}+r \bar{P}_{x} \bar{E}_{0}
$$

where $r$ is small enough such that $P_{y}(1,1)-r \geq 0[16,17]$. If we choose $r$ such that strict inequality holds, the remainder $\tilde{P}$ is still a full norm. Note that $r$ is proportional to $\Delta y$. The dissipation term can thus be rewritten as

$$
\begin{aligned}
D I & =2 \varepsilon\left[\begin{array}{c}
\bar{D}_{x} w \\
\bar{D}_{y} w
\end{array}\right]^{T}\left[\begin{array}{cc}
\tilde{P} & 0 \\
0 & \tilde{P}
\end{array}\right]\left[\begin{array}{ll}
\bar{C}_{11} & \bar{C}_{12} \\
\bar{C}_{21} & \bar{C}_{22}
\end{array}\right]\left[\begin{array}{l}
\bar{D}_{x} w \\
\bar{D}_{y} w
\end{array}\right] \\
& +2 \varepsilon r\left[\begin{array}{c}
\bar{D}_{x} w \\
\bar{D}_{y} w
\end{array}\right]^{T}\left[\begin{array}{cc}
\bar{P}_{x} \bar{E}_{0} & 0 \\
0 & \bar{P}_{x} \bar{E}_{0}
\end{array}\right]\left[\begin{array}{ll}
\bar{C}_{11} & \bar{C}_{12} \\
\bar{C}_{21} & \bar{C}_{22}
\end{array}\right]\left[\begin{array}{c}
\bar{D}_{x} w \\
\bar{D}_{y} w
\end{array}\right] .
\end{aligned}
$$

The second term in (43) can be used to fill in the empty $2 \times 2$ bottom block in $\bar{M}_{0}$ to obtain

$$
\bar{M}=\left[\begin{array}{ccc}
\bar{m}_{1} & \bar{m}_{2} & \bar{m}_{3} \\
\bar{m}_{2}^{T} & 2 r \bar{C}_{11} & 2 r \bar{C}_{12} \\
\bar{m}_{3} & 2 r \bar{C}_{21} & 2 r \bar{C}_{22}
\end{array}\right]
$$

To determine positive semi-definiteness of $\bar{M}$ it is sufficient to only consider the reduced matrix

$$
M=\left[\begin{array}{ccc}
-2\left(\sigma_{2} \alpha H_{2}+\sigma_{3} H_{3}+\sigma_{4} \phi H_{4}\right) & C_{21}-\Theta & C_{22}+\sigma_{2} \beta H_{2}+\sigma_{4} \psi H_{4} \\
C_{21}-\Theta^{T} & 2 r C_{11} & 2 r C_{12} \\
C_{22}+\sigma_{2} \beta H_{2}+\sigma_{4} \psi H_{4} & 2 r C_{21} & 2 r C_{22}
\end{array}\right]
$$

where we have removed the Kronecker products. This can be done since the Kronecker product is permutation similar, i.e. there exist a permutation matrix $Y$ such that for arbitrary square matrices $A$ and $B$ we have $A \otimes B=Y^{T}(B \otimes A) Y$. Hence we can rewrite (38) as

$$
\|w\|_{t}^{2}=-\varepsilon(Y W)^{T}\left(M \otimes P_{0}\right) Y W-\tilde{\mathrm{DI}}
$$

where $P_{0}=P_{x} \otimes E_{0}$ is positive semi-definite.

In order to proceed we chose

$$
\Theta=\left[\begin{array}{cccc}
0 & 0 & 0 & 0 \\
0 & 0 & \frac{\lambda+\mu}{2 \bar{\rho}} & 0 \\
0 & 0 & 0 & 0 \\
0 & 0 & 0 & 0
\end{array}\right]
$$


The matrix $M$ in (45) is of size $12 \times 12$ but with the 1 st, 5 th and 9 th row and column being zero. We can hence remove these rows and columns and condense (45) into the $9 \times 9$ matrix

$$
\tilde{M}=\left[\begin{array}{ccc}
-2\left(\sigma_{2} \alpha \tilde{H}_{2}+\sigma_{3} \tilde{H}_{3}+\sigma_{4} \phi \tilde{H}_{4}\right) & \tilde{C}_{21}-\tilde{\Theta} \tilde{C}_{22}+\sigma 2 \beta \tilde{H}_{2}+\sigma_{4} \psi \tilde{H}_{4} \\
\tilde{C}_{21}-\tilde{\Theta}^{T} & 2 r \tilde{C}_{11} & 2 r \tilde{C}_{12} \\
\tilde{C}_{22}+\sigma_{2} \alpha \tilde{H}_{2}+\sigma_{4} \psi \tilde{H}_{4} & 2 r \tilde{C}_{21} & 2 r \tilde{C}_{22}
\end{array}\right] .
$$

By defining the matrices

$$
\begin{aligned}
\tilde{A} & =\sigma_{2} \alpha \tilde{H}_{2}+\sigma_{3} \tilde{H}_{3}+\sigma_{4} \phi \tilde{H}_{4}, \\
\tilde{B} & =\tilde{C}_{22}+\sigma_{2} \beta \tilde{H}_{2}+\sigma_{4} \psi \tilde{H}_{4}, \\
\tilde{C} & =\left[\begin{array}{ll}
\tilde{C}_{11} & \tilde{C}_{12} \\
\tilde{C}_{21} & \tilde{C}_{22}
\end{array}\right], \\
\tilde{J} & =\left[\begin{array}{lll}
\tilde{C}_{21}-\tilde{\Theta} & \tilde{B}
\end{array}\right]
\end{aligned}
$$

we can rewrite (48) as

$$
\tilde{M}=\left[\begin{array}{cc}
-2 \tilde{A} & \tilde{J} \\
\tilde{J}^{T} & 2 r \tilde{C}
\end{array}\right]
$$

which can be rotated into block-dagonal form. The rotation matrix is defined by

$$
\tilde{S}=\left[\begin{array}{cc}
I_{3} & -\frac{1}{2 r} \tilde{J} \tilde{C}^{-1} \\
0_{6 \times 3} & I_{6}
\end{array}\right]
$$

where $0_{p \times q}$ is a zero matrix of size indicated by the subscript. Note that $\tilde{C}^{-1}$ is well-defined since we have removed the zero rows and columns. Using (51) we can rotate $(50)$ by

$$
\tilde{S} \tilde{M} \tilde{S}^{T}=\left[\begin{array}{cc}
-2 \tilde{A}-\frac{1}{2 r} \tilde{J} \tilde{C}^{-1} \tilde{J}^{T} & 0_{3 \times 6} \\
0_{6 \times 3} & 2 r \tilde{C}
\end{array}\right]
$$

and it is clear that a sufficient condition for positive semi-definiteness is that the Schur complement of $2 r \tilde{C}$ in $\tilde{M}$ satisfies

$$
Q=-2 \tilde{A}-\frac{1}{2 r} \tilde{J} \tilde{C}^{-1} \tilde{J}^{T} \geq 0 .
$$

Equation (53) leads to the main result of this paper which is

Theorem 2.3. The scheme for the compressible Navier-Stokes equations

$$
w_{t}+\bar{D}_{x} \mathbf{F}+\bar{D}_{y} \mathbf{G}=\mathbb{S}
$$

with Robin boundary conditions given in (26) and (27), where $\mathbb{S}$ is given by (28), can be made stable for all choices of $\alpha, \beta, \phi$ and $\psi$ using (36), (47) and appropriate choices of $\sigma_{2,3,4}$.

Proof. The inviscid part that implements the wall normal velocity boundary condition for the Euler equations is bounded using (36). Using (47), the matrix $Q$ in (53) is a $3 \times 3$ diagonal matrix

$$
Q=\left[\begin{array}{ccc}
\lambda_{1}\left(\sigma_{2}\right) & 0 & 0 \\
0 & \lambda_{2}\left(\sigma_{3}\right) & 0 \\
0 & 0 & \lambda_{3}\left(\sigma_{4}\right)
\end{array}\right]
$$


where the diagonal entries are given by

$$
\begin{aligned}
& \lambda_{1}\left(\sigma_{2}\right)=-2 \sigma_{2} \alpha-\frac{2 \mu\left(\mu+\sigma_{2} \beta \bar{\rho}\right)^{2}}{r(\lambda+3 \mu)(\mu-\lambda) \bar{\rho}}, \\
& \lambda_{2}\left(\sigma_{3}\right)=-2 \sigma_{3}-\frac{1}{2} \frac{\lambda+2 \mu}{r \bar{\rho}}, \\
& \lambda_{3}\left(\sigma_{4}\right)=-2 \sigma_{4} \phi-\frac{1}{2} \frac{\left(\gamma \mu+\sigma_{4} \psi \operatorname{Pr} \bar{\rho}\right)^{2}}{r \gamma \mu \operatorname{Pr} \bar{\rho}} .
\end{aligned}
$$

For any choice of $\alpha, \beta, \phi$ and $\psi$ such that no boundary condition is removed and (15) holds, it is possible to determine $\sigma_{2,3,4}$ such that $\lambda_{1,2,3} \geq 0$. The actual values of $\sigma_{2,3,4}$ are determined once the choices of $\alpha, \beta, \phi$ and $\psi$ has been made.

The standard no-slip boundary conditions with prescribed temperature

$$
u=0, \quad v=0, \quad T=T_{w}
$$

where $T_{w}$ is the wall temperature follows as a corollary.

Corollary 2.4. The standard no-slip boundary conditions with prescribed temperature given by

$$
u=0, \quad v=0, \quad T=T_{w}
$$

are stable using (36), (47) and

$$
\begin{aligned}
\sigma_{2} & \leq-\frac{\mu^{3}}{r(\lambda+3 \mu)(\mu-\lambda) \bar{\rho}} \\
\sigma_{3} & \leq-\frac{1}{4} \frac{\lambda+2 \mu}{r \bar{\rho}} \\
\sigma_{4} & \leq-\frac{1}{4 r} \frac{\gamma \mu}{\operatorname{Pr} \bar{\rho}} .
\end{aligned}
$$

Proof. The no-slip boundary conditions with prescribed temperature, which are thoroughly discussed in [2], are obtained by putting

$$
\alpha=1, \quad \beta=0, \quad \phi=1, \quad \psi=0
$$

in which case $(56)$ reduces to

$$
\begin{aligned}
& \lambda_{1}\left(\sigma_{2}\right)=-2 \sigma_{2}-\frac{2 \mu^{3}}{r(\lambda+3 \mu)(\lambda-\mu) \bar{\rho}}, \\
& \lambda_{2}\left(\sigma_{3}\right)=-2 \sigma_{3}-\frac{1}{2} \frac{\lambda+2 \mu}{r \bar{\rho}}, \\
& \lambda_{3}\left(\sigma_{4}\right)=-2 \sigma_{4}-\frac{1}{2} \frac{\gamma \mu}{r \operatorname{Pr} \bar{\rho}} .
\end{aligned}
$$

By demanding

$$
\lambda_{i} \geq 0, \quad i=1,2,3
$$

we obtain (59). 
Note that the estimates (59) are sharp since there are no approximations or embeddings involved in the derivation of (53) as in contrast to the result in [2]. The results in [2] are obtained in this setting by having

$$
\Theta=0_{4 \times 4}
$$

and taking

$$
\sigma_{1,2,3}=\sigma \leq-\frac{1}{4 r} \lambda_{\max }
$$

where $\lambda_{\max }$ is the maximum eigenvalue of $\tilde{J} \tilde{C}^{-1} \tilde{J}^{T}$. Since the system becomes stiffer with increasing magnitude of the coefficients it is desirable with sharp estimates to minimize the magnitudes. If we compare (59) and (64) we get

$$
\begin{aligned}
\frac{\sigma_{2}}{\sigma} & =\frac{4 \mu^{2} P r}{\gamma(\lambda+3 \mu)(\mu-\lambda)}, \\
\frac{\sigma_{3}}{\sigma} & =\frac{(\lambda+2 \mu) \operatorname{Pr}}{\gamma \mu} \\
\frac{\sigma_{4}}{\sigma} & =1 .
\end{aligned}
$$

With some reasonable numerical values, $\rho=1, \gamma=1.4, \operatorname{Pr}=0.72, \mu=1$ and $\lambda=-\frac{2}{3} \mu$, the ratios become

$$
\frac{\sigma_{2}}{\sigma} \approx 0.53, \quad \frac{\sigma_{3}}{\sigma} \approx 0.69, \quad \frac{\sigma_{4}}{\sigma}=1
$$

which is an improvement for the velocity components.

The proof of stability using (63) and (64) does not extend to the case where $\beta \neq 0$ in which case $\Theta \neq 0_{4 \times 4}$ is required.

For the adiabatic solid wall boundary conditions we have

Corollary 2.5. The adiabatic boundary conditions

$$
u=0, \quad v=0, \quad T_{y}=0
$$

are stable using (36), (47) and

$$
\begin{aligned}
\sigma_{2} & \leq-\frac{\mu^{3}}{r(\lambda+3 \mu)(\mu-\lambda) \bar{\rho}}, \\
\sigma_{3} & \leq-\frac{1}{4} \frac{\lambda+2 \mu}{r \bar{\rho}}, \\
\sigma_{4} & =-\frac{\gamma \mu}{\operatorname{Pr} \bar{\rho}} .
\end{aligned}
$$

Proof. The adiabatic boundary conditions are obtained by having

$$
\alpha=1, \quad \beta=0, \quad \phi=0, \quad \psi=1
$$

in which case $(56)$ reduces to

$$
\begin{aligned}
& \lambda_{1}\left(\sigma_{2}\right)=-2 \sigma_{2}-\frac{2 \mu^{3}}{r(\lambda+3 \mu)(\lambda-\mu) \bar{\rho}}, \\
& \lambda_{2}\left(\sigma_{3}\right)=-2 \sigma_{3}-\frac{1}{2} \frac{\lambda+2 \mu}{r \bar{\rho}}, \\
& \lambda_{3}\left(\sigma_{4}\right)=-\frac{1}{2} \frac{\left(\gamma \mu+\sigma_{4} \operatorname{Pr} \bar{\rho}\right)^{2}}{r \gamma \mu \operatorname{Pr} \bar{\rho}} .
\end{aligned}
$$


By demanding

$$
\lambda_{i} \geq 0, \quad i=1,2,3
$$

we obtain (68).

Remember that $r$ is proportional to $\Delta y$. As the mesh is refined, the penalty coefficients will increase in magnitude and make the discretization stiffer. If $\beta, \psi \neq 0$ we can cancel the $1 / r$ dependence in $\sigma_{2,4}$ by choosing

$$
\sigma_{2}=-\frac{1}{\beta} \frac{\mu}{\bar{\rho}}, \quad \sigma_{4}=-\frac{1}{\psi} \frac{\gamma \mu}{\operatorname{Pr} \bar{\rho}}
$$

in which case $(56)$ reduces to

$$
\begin{aligned}
\lambda_{1} & =\frac{2 \mu}{\bar{\rho}} \frac{\alpha}{\beta}, \\
\lambda_{2}\left(\sigma_{3}\right) & =-2 \sigma_{3}-\frac{1}{2} \frac{\lambda+2 \mu}{r \bar{\rho}}, \\
\lambda_{3} & =\frac{2 \gamma \mu}{\operatorname{Pr} \bar{\rho}} \frac{\phi}{\psi} .
\end{aligned}
$$

It is easy to see from (73) that the continuous well-posedness conditions (15) are required in order for $\lambda_{1,3} \geq 0$. The $1 / r$ dependence in $\sigma_{3}$ is not possible to remove unless a different form of the SAT is used.

Remark 2.6. For the north boundary at $y=1$, the conditions in Theorem 2.3 and its corollaries apply without modifications. However, the Robin boundary conditions (26) are replaced by

$$
\alpha u+\beta u_{y}=g_{1} \quad \phi T+\psi T_{y}=g_{2} \quad v=g_{3} .
$$

\section{Numerical results}

The stability theory developed in the previous section does not depend on the order of accuracy of the numerical scheme. In order to verify that the scheme attains its design order we will use the method of manufactured solutions.

Any sufficiently smooth function $H(x, y, t)$ is a solution to the modified NavierStokes equations

$$
q_{t}+F_{x}+G_{y}=R(x, y, t)
$$

where the forcing function $R(x, y, t)$ has to be appropriately chosen depending on $H(x, y, t)$. By the principle of Duhamel [18], the inhomogeneous equation (75) is well-posed if the homogeneous equation (1) is [18]. The boundary conditions remain unchanged and we can use the manufactured solution $H(x, y, t)$ to create the initial and boundary data. Thus we have an analytic solution to (75) which can be used to test the order of accuracy of the computational scheme.

In this particular case we specify

$$
\begin{aligned}
& \rho(x, y, t)=e^{-\nu(\sin (\xi \pi x-t))^{2}-\nu(\cos (\xi \pi y-t))^{2}} \\
& u(x, y, t)=\cos (\xi \pi(x+y)-t) \\
& v(x, y, t)=\sin (\xi \pi(x+y)-t) \\
& p(x, y, t)=e^{-\nu(\sin (\xi \pi(x-y)-t))^{2}}
\end{aligned}
$$


Table 1: Order of accuracy

\begin{tabular}{|c|c|c|c|c|}
\hline \# grid points & $32 \times 32$ & $64 \times 64$ & $128 \times 128$ & $256 \times 256$ \\
\hline \multicolumn{5}{|c|}{ 2nd-order } \\
\hline$\rho$ & 1.5034 & 1.7651 & 1.9666 & 1.9752 \\
\hline$\rho u$ & 1.8596 & 2.0101 & 2.0594 & 2.0402 \\
\hline$\rho v$ & 1.8540 & 2.0216 & 2.0643 & 2.0163 \\
\hline$e$ & 1.4702 & 1.8064 & 2.0253 & 1.9725 \\
\hline \multicolumn{5}{|c|}{ 4th-order } \\
\hline$\rho$ & 2.1722 & 2.3449 & 2.7873 & 2.7933 \\
\hline$\rho u$ & 2.5558 & 2.6331 & 2.7796 & 2.6916 \\
\hline$\rho v$ & 2.5409 & 2.5925 & 2.8033 & 2.7474 \\
\hline$e$ & 2.1944 & 2.4076 & 2.7627 & 2.7141 \\
\hline \multicolumn{5}{|c|}{ 6th-order } \\
\hline$\rho$ & 3.4865 & 3.6814 & 3.8377 & 3.8038 \\
\hline$\rho u$ & 3.7509 & 3.7349 & 3.9500 & 3.9811 \\
\hline$\rho v$ & 3.6202 & 3.8639 & 4.0055 & 4.0174 \\
\hline$e$ & 3.4023 & 4.0812 & 4.1063 & 3.9139 \\
\hline
\end{tabular}

where $\nu$ and $\xi$ can be used to tune the amplitude and frequency of the solution. In this case we have chosen $\nu=\xi=0.1$. Using (76) we specify $H(x, y, t)$ as

$$
H(x, y, t)=\left[\begin{array}{c}
\rho \\
\rho u \\
\rho v \\
e
\end{array}\right], \quad e=\frac{p}{\gamma-1}+\frac{1}{2} \rho\left(u^{2}+v^{2}\right)
$$

where $\gamma=1.4$.

The scheme for $(75)$ is

$$
w_{t}+\bar{D}_{x} \mathbf{F}+\bar{D}_{y} \mathbf{G}=R(x, y, t)+\mathbb{S}
$$

and in order to obtain a higher order accurate scheme, the difference operators $\bar{D}_{x, y}$ are simply replaced with operators of the desired order of accuracy. The penalty coefficients in Theorem 2.3 remain unchanged. The forcing function $R(x, y, t)$ is too tedious to write in text but can be computed using a symbolic software such as Maple ${ }^{\circledR}$.

The scheme (78) was implemented using SBP operators of order 2, 4 and 6 which gives a global accuracy of 2,3 and $4[10,19]$. The result can be seen in Table 1 . The order of accuracy is independent of the choices of $\alpha, \beta, \phi$ and $\psi$ and in Table 1 the no-slip with prescribed temperature, using $\alpha=1, \beta=0, \phi=1$ and $\psi=0$, is seen.

\section{Applications}

An application of the Robin boundary condition is the slip-flow boundary conditions used for moderate Knudsen numbers $(K n)$ in micro fluid flows. The slip-flow boundary conditions extends the use of the Navier-Stokes equations to the slip-flow regime where $10^{-3} \leq K n \leq 10^{-1}[20]$. 
Computations in the slip-flow regime corresponds to having $\alpha=1, \phi=1, \psi=0$ and $\beta=K n$ which gives a first order slip-flow boundary condition. Stability is shown in

Corollary 4.1. The first order slip-flow boundary conditions

$$
u=(K n) u_{y}, \quad v=0, \quad T=T_{w}
$$

are stable using (36), (47) and

$$
\begin{aligned}
\sigma_{2} & =-\frac{\mu}{(K n) \bar{\rho}} \\
\sigma_{3} & \leq-\frac{1}{4} \frac{\lambda+2 \mu}{r \bar{\rho}}, \\
\sigma_{4} & \leq-\frac{1}{4 r} \frac{\gamma \mu}{\operatorname{Pr} \bar{\rho}} .
\end{aligned}
$$

Proof. The slip-flow boundary conditions are obtained by

$$
\alpha=1, \quad \beta=K n, \quad \phi=1, \quad \psi=0
$$

in which case $(56)$ reduces to

$$
\begin{aligned}
& \lambda_{1}\left(\sigma_{2}\right)=-2 \sigma_{2}-\frac{2 \mu\left(\mu+\sigma_{2}(K n) \bar{\rho}\right)^{2}}{r(\lambda+3 \mu)(\lambda-\mu) \bar{\rho}}, \\
& \lambda_{2}\left(\sigma_{3}\right)=-2 \sigma_{3}-\frac{1}{2} \frac{\lambda+2 \mu}{r \bar{\rho}}, \\
& \lambda_{3}\left(\sigma_{4}\right)=-2 \sigma_{4}-\frac{1}{2} \frac{\gamma \mu}{r \operatorname{Pr} \bar{\rho}} .
\end{aligned}
$$

By demanding

$$
\lambda_{i} \geq 0, \quad i=1,2,3
$$

we obtain (80).

Figures 1 to 4 shows the flow field from no-slip $(\beta=0)$ to almost full slip $(\beta=1)$. In the computations we have used the domain $0 \leq x \leq 5,0 \leq y \leq 1$ with $512 \times 128$ grid points. The Mach number is 0.5 and the Reynolds number is 100. All scales are normalized with respect to the no-slip case.

The inflow and outflow boundary conditions are implemented as described in [1] which means that there is a severe missmatch between the boundary conditions and the boundary data at the corners. However because of the weak boundary treatment the computations remain stable. 


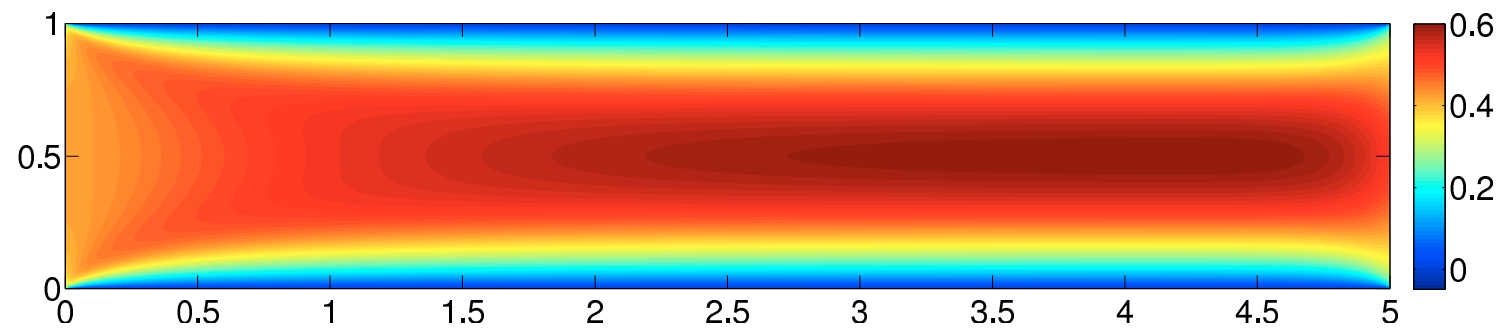

(a) Momentum

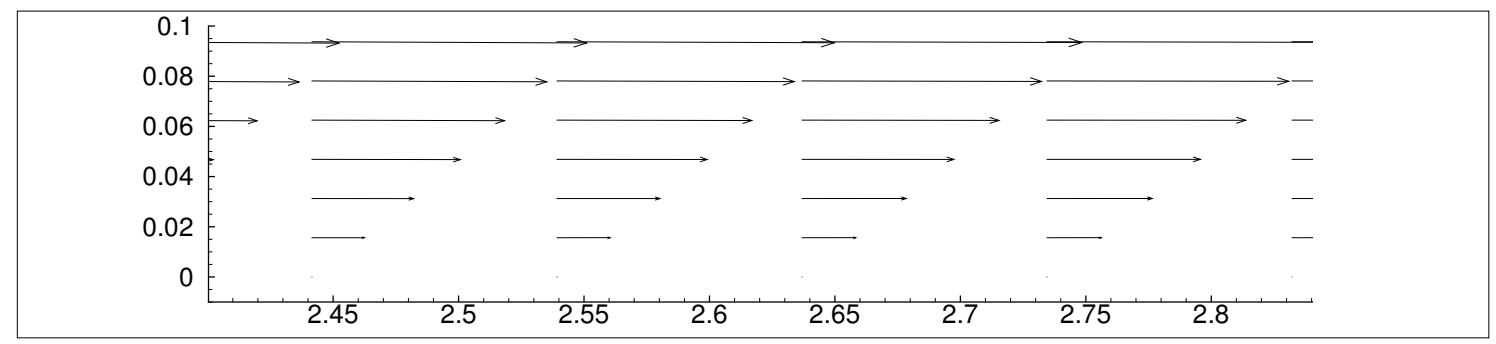

(b) Close-up of momentum field at the center of the south boundary

Figure 1: $\beta=0.0$, corresponding to no-slip

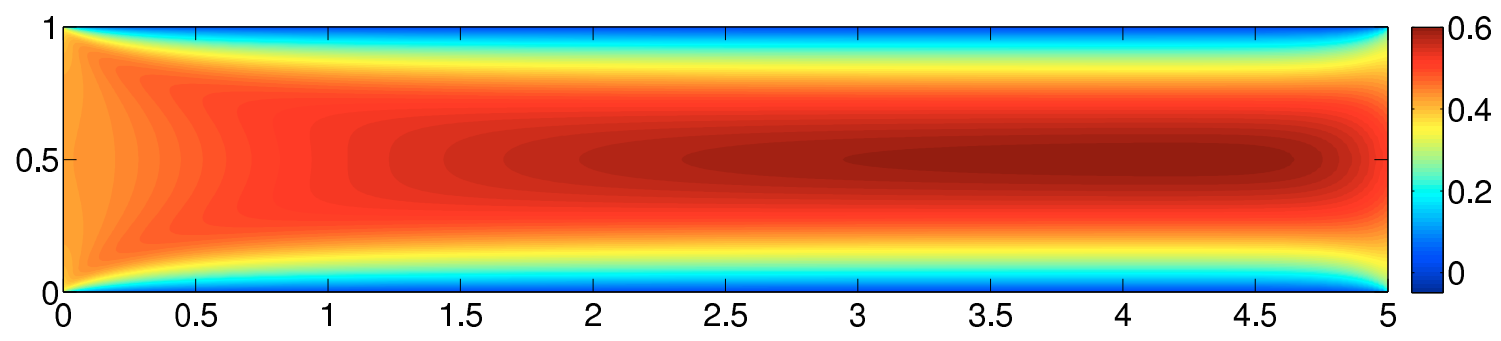

(a) Momentum

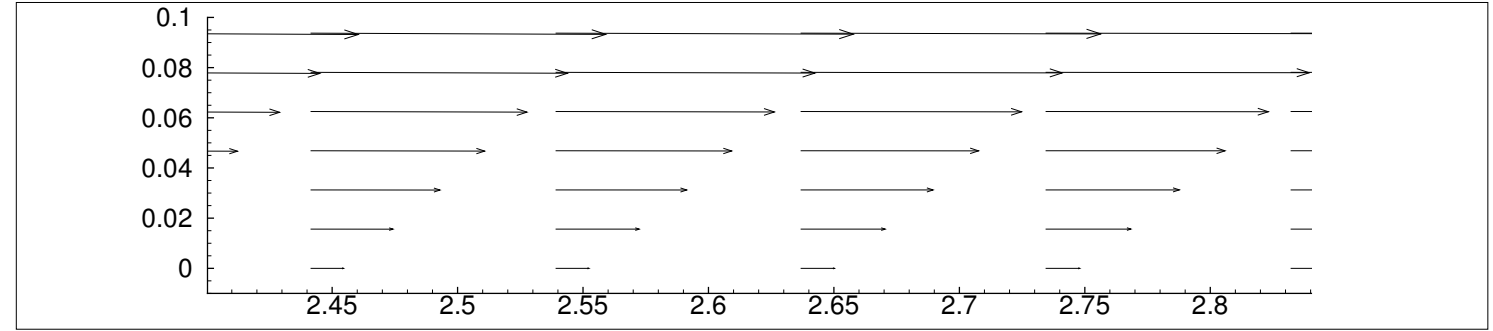

(b) Close-up of momentum field at the center of the south boundary

Figure 2: $\beta=0.01$, corresponding to moderate slip 


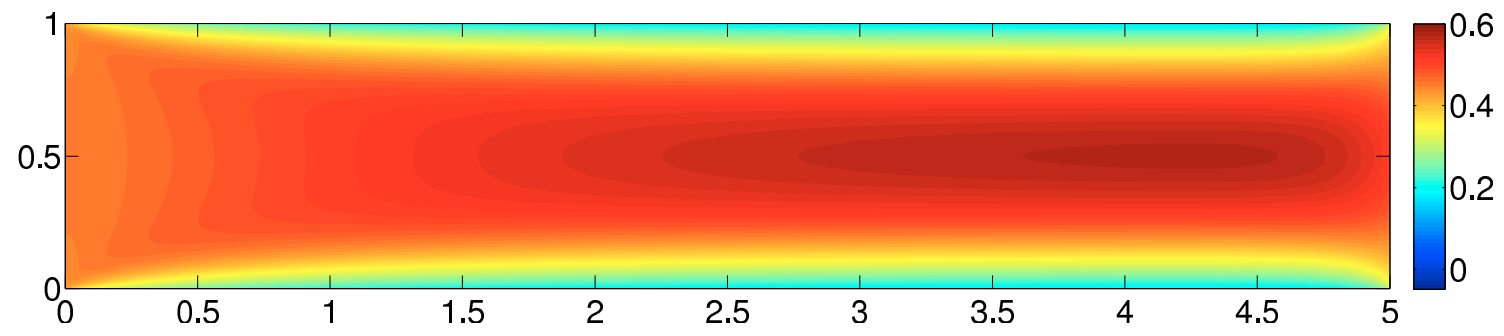

(a) Momentum

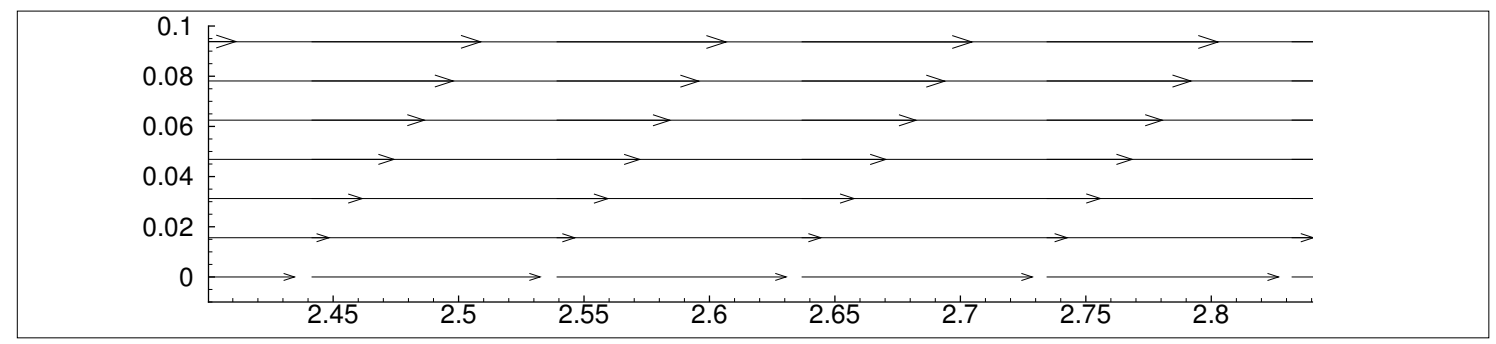

(b) Close-up of momentum field at the center of the south boundary

Figure 3: $\beta=0.1$, corresponding to large slip

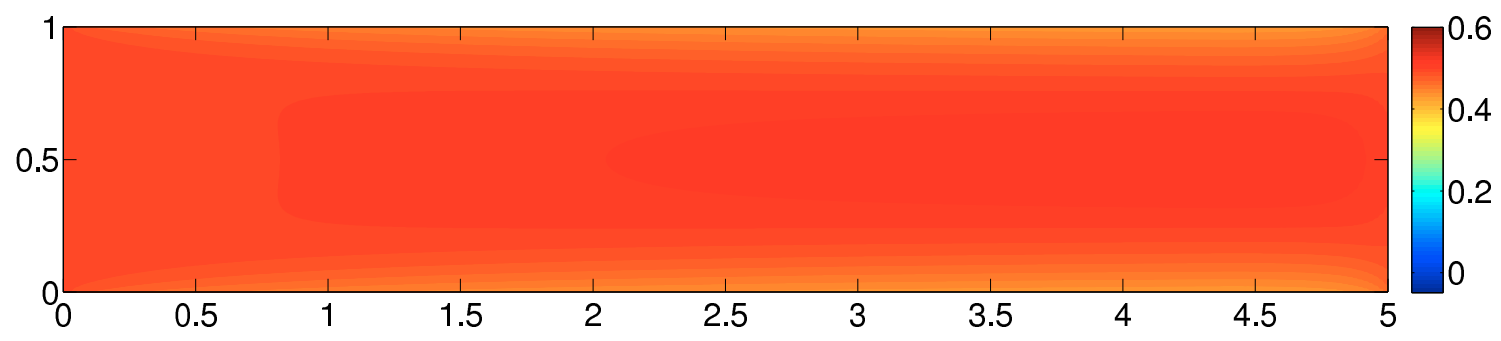

(a) Momentum

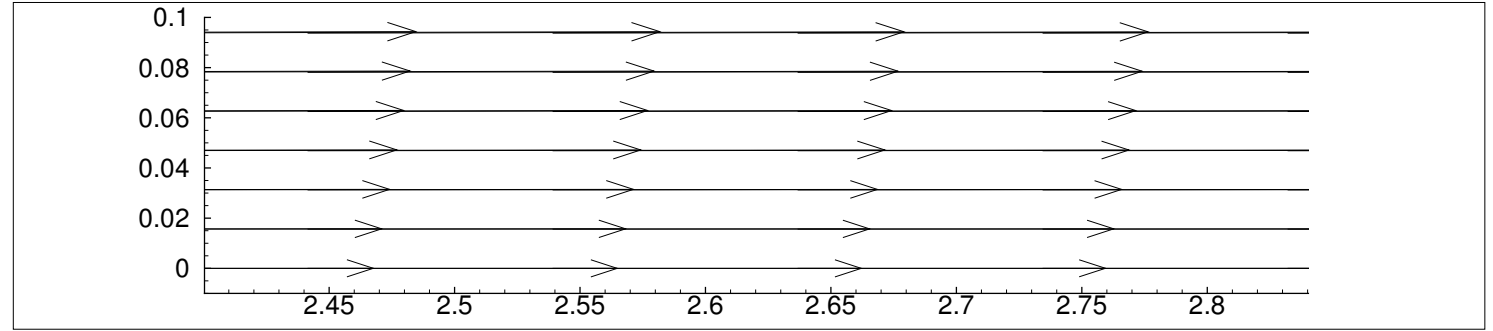

(b) Close-up of momentum field at the center of the south boundary

Figure 4: $\beta=1.0$, corresponding to almost full slip

\section{Summary and conclusions}

We have proved stability for Robin solid wall boundary conditions for the compressible Navier-Stokes equations using a finite difference method on SummationBy-Parts (SBP) form with weak boundary conditions using the Simultaneous Approximation Term (SAT).

The formulation of the SAT allows for easy change between common boundary conditions such as the no-slip with prescribed temperature or temperature gradient and slip-flow or any combination thereof. 
The energy estimates were derived without using approximations or embeddings which yields sharp estimates in contrast to previous results.

The accuracy of the numerical scheme was tested using a manufactured solution. The computational scheme was verified to attain 2nd-, 3rd- and 4th-order of accuracy which are the design orders of the SBP scheme.

We did computations of flows in a rectangular domain when the solid wall boundary conditions were changed from no-slip to substantial slip by a simple variation of one parameter.

\section{Acknowledgments}

The computations were performed on resources provided by SNIC through Uppsala Multidisciplinary Center for Advanced Computational Science (UPPMAX) under Project p2010056. The reviewer of the manuscript is greatly acknowledged for improving the quality of the final article.

\section{Appendix A. Matrix coefficients}

The matrix coefficients in (6) are given by

$$
\begin{array}{ccc}
A= & {\left[\begin{array}{cccc}
\bar{u} & \frac{\bar{c}}{\sqrt{\gamma}} & 0 & 0 \\
\overline{\bar{c}} & \bar{u} & 0 & \bar{c} \sqrt{\frac{\gamma-1}{\gamma}} \\
\frac{\sqrt{\gamma}}{0} & 0 & \bar{u} & 0 \\
0 & \bar{c} \sqrt{\frac{\gamma-1}{\gamma}} & 0 & \bar{u}
\end{array}\right], \quad B=\left[\begin{array}{cccc}
\bar{v} & 0 & \frac{\bar{c}}{\sqrt{\gamma}} & 0 \\
0 & \bar{v} & 0 & 0 \\
\bar{c} & 0 & \bar{v} & \bar{c} \sqrt{\frac{\gamma-1}{\gamma}} \\
\overline{\sqrt{\gamma}} & 0 & \bar{c} \sqrt{\frac{\gamma-1}{\gamma}} & \bar{v}
\end{array}\right]} \\
0 & 0 & \\
C_{11} & =\left[\begin{array}{cccc}
0 & 0 & 0 & 0 \\
0 & \frac{\lambda+2 \mu}{\bar{\rho}} & 0 & 0 \\
0 & 0 & \frac{\mu}{\bar{\rho}} & 0 \\
0 & 0 & 0 & \frac{\gamma \mu}{\operatorname{Pr} \bar{\rho}}
\end{array}\right], \quad C_{22}=\left[\begin{array}{cccc}
0 & 0 & 0 & 0 \\
0 & \frac{\mu}{\bar{\rho}} & 0 & 0 \\
0 & 0 & \frac{\lambda+2 \mu}{\bar{\rho}} & 0 \\
0 & 0 & 0 & \frac{\gamma \mu}{\operatorname{Pr} \bar{\rho}}
\end{array}\right], \\
C_{12} & =C_{21}=\left[\begin{array}{cccc}
0 & 0 & 0 & 0 \\
0 & 0 & \frac{\lambda+\mu}{2 \bar{\rho}} & 0 \\
0 & \frac{\lambda+\mu}{2 \bar{\rho}} & 0 & 0 \\
0 & 0 & 0 & 0
\end{array}\right] .
\end{array}
$$

\section{Appendix B. SBP operators}

In the second order case the SBP operators are explicitly given by

$$
D_{\xi}=P_{\xi}^{-1} Q_{\xi}
$$


where $\xi$ is either $x$ or $y$ and

$$
\begin{aligned}
& P_{\xi}=\frac{1}{\Delta \xi}\left[\begin{array}{ccccc}
\frac{1}{2} & 0 & 0 & \ldots & 0 \\
0 & 1 & 0 & \ldots & 0 \\
\vdots & \vdots & \ddots & \vdots & \vdots \\
0 & \ldots & 0 & 1 & 0 \\
0 & \ldots & 0 & 0 & \frac{1}{2}
\end{array}\right], Q_{\xi}=\frac{1}{2}\left[\begin{array}{ccccccc}
-1 & 1 & 0 & 0 & \ldots & 0 & 0 \\
-1 & 0 & 1 & 0 & \ldots & 0 & 0 \\
0 & -1 & 0 & 1 & 0 & \ldots & 0 \\
\vdots & \ddots & \ddots & \ddots & \ddots & \ddots & \vdots \\
0 & \ldots & 0 & -1 & 0 & 1 & 0 \\
0 & \ldots & 0 & 0 & -1 & 0 & 1 \\
0 & \ldots & 0 & 0 & 0 & -1 & 1
\end{array}\right] \\
& D_{\xi}=\frac{1}{2 \Delta \xi}\left[\begin{array}{ccccccc}
-2 & 2 & 0 & 0 & \ldots & 0 & 0 \\
-1 & 0 & 1 & 0 & \ldots & 0 & 0 \\
0 & -1 & 0 & 1 & 0 & \ldots & 0 \\
\vdots & \ddots & \ddots & \ddots & \ddots & \ddots & \vdots \\
0 & \ldots & 0 & -1 & 0 & 1 & 0 \\
0 & \ldots & 0 & 0 & -1 & 0 & 1 \\
0 & \ldots & 0 & 0 & 0 & -2 & 2
\end{array}\right]
\end{aligned}
$$

For SBP operators of higher order accuracy we refer the reader to $[10,11]$.

\section{References}

[1] Magnus Svärd, Mark H. Carpenter, and Jan Nordström. A stable high-order finite difference scheme for the compressible Navier-Stokes equations, far-field boundary conditions. Journal of Computational Physics, 225(1):1020-1038, 2007.

[2] Magnus Svärd and Jan Nordström. A stable high-order finite difference scheme for the compressible Navier-Stokes equations: No-slip wall boundary conditions. Journal of Computational Physics, 227(10):4805 - 4824, 2008.

[3] Jan Nordström, Jing Gong, Edwin van der Weide, and Magnus Svärd. A stable and conservative high order multi-block method for the compressible NavierStokes equations. Journal of Computational Physics, 228(24):9020-9035, 2009.

[4] Jan Nordström and Magnus Svärd. Well-Posed Boundary Conditions for the Navier-Stokes Equations. SIAM Journal on Numerical Analysis, 43(3):12311255, 2005.

[5] Ken Mattsson, Magnus Svärd, and Mohammad Shoeybi. Stable and accurate schemes for the compressible Navier-Stokes equations. Journal of Computational Physics, 227:2293-2316, February 2008.

[6] Magnus Svärd, Ken Mattsson, and Jan Nordström. Steady-State Computations Using Summation-by-Parts Operators. Journal of Scientific Computing, 24(1):79-95, 2005.

[7] Ken Mattsson, Magnus Svärd, Mark Carpenter, and Jan Nordström. Highorder accurate computations for unsteady aerodynamics. Computers and Flu$i d s, 36(3): 636-649,2007$. 
[8] X. Huan, J.E. Hicken, and D.W. Zingg. Interface and Boundary Schemes for High-Order Methods. In the 39th AIAA Fluid Dynamics Conference, AIAA Paper No. 2009-3658, San Antonio, USA, 22-25 June 2009.

[9] Jan Nordström, Sofia Eriksson, Craig Law, and Jing Gong. Shock and vortex calculations using a very high order accurate Euler and Navier-Stokes solver. Journal of Mechanics and MEMS, 1(1):19-26, 2009.

[10] Bo Strand. Summation by Parts for Finite Difference Approximations for d/dx. Journal of Computational Physics, 110(1):47 - 67, 1994.

[11] Ken Mattsson and Jan Nordström. Summation by parts operators for finite difference approximations of second derivatives. Journal of Computational Physics, 199(2):503-540, 2004.

[12] Ken Mattsson. Summation by parts operators for finite difference approximations of second-derivatives with variable coefficients. Technical Report 2010-023, Uppsala University, Division of Scientific Computing, 2010.

[13] Qaisar Abbas and Jan Nordström. Weak versus strong no-slip boundary conditions for the Navier-Stokes equations. Engineering Applications of Computational Fluid Mechanics, 4:29-38, 2010.

[14] Saul Abarbanel and David Gottlieb. Optimal time splitting for two- and threedimensional Navier-Stokes equations with mixed derivatives. Journal of Computational Physics, 41(1):1 - 33, 1981.

[15] T. H. Pulliam and D. S. Chaussee. A diagonal form of an implicit approximatefactorization algorithm. Journal of Computational Physics, 39(2):347 - 363, 1981.

[16] Mark H. Carpenter, Jan Nordström, and David Gottlieb. A Stable and Conservative Interface Treatment of Arbitrary Spatial Accuracy. Journal of Computational Physics, 148(2):341 - 365, 1999.

[17] Mark H. Carpenter, Jan Nordström, and David Gottlieb. Revisiting and extending interface penalties for multi-domain summation-by-parts operators. Journal of Scientific Computing, 45(1-3):118-150, 2010.

[18] Bertil Gustafsson, Heinz-Otto Kreiss, and Joseph Oliger. Time Dependent Problems and Difference Methods. Wiley Interscience, 1995.

[19] Magnus Svärd and Jan Nordström. On the order of accuracy for difference approximations of initial-boundary value problems. Journal of Computational Physics, 218(1):333-352, 2006.

[20] Mohamed Gad el Hak. The fluid mechanics of microdevices - the freeman scholar lecture. Journal of Fluids Engineering, 121(1):5-33, 1999. 